\title{
Stationary Equilibria in Discounted Stochastic Games with Weakly Interacting Players
}

\author{
ULRICH HORST* \\ Institut für Mathematik - Bereich Stochastik \\ Humboldt-Universität zu Berlin \\ Unter den Linden 6 \\ 10099 Berlin \\ Germany \\ E-mail:horst@mathematik.hu-berlin.de \\ Fax: +49 (0) 3020935848
}

3rd October 2002

\begin{abstract}
We give sufficient conditions for a non-zero sum discounted stochastic game with compact and convex action spaces and with norm-continuous transition probabilities, but with possibly unbounded state space, to have a Nash equilibrium in homogeneous Markov strategies that depends in a Lipschitz continuous manner on the current state. If the underlying state space is compact this yields the existence of a stationary equilibrium. For a special class of stochastic games which arise in microstructure models for financial markets we establish the existence of equilibria which guarantee that the state sequence converges in distribution to a unique stationary measure.
\end{abstract}

JEL subject classification. D81, E32

Key words and phrases. Stochastic Games, Stationary Equilibria, Microstructure Models for Financial Markets

\footnotetext{
${ }^{*}$ This work was initiated while I was visiting Bendheim Center for Finance, Princeton University. I would like to thank Jose Scheinkman for many valuable discussions. Financial support through the German Academic Exchange Service (DAAD) and through the NSF grant No. 0001647 to Princeton University is gratefully acknowledged.
} 


\section{Introduction}

This paper considers infinite horizon discounted non-zero sum stochastic games with compact and convex action spaces and with norm-continuous transition probabilities. We formulate conditions on the utility functions and on the law of motion which guarantee the existence of stationary equilibria in Markov strategies, i.e., of Markov equilibria which have the additional property that the equilibrium process admits a stationary measure in the sense of Duffie, Geanakopolos, MasColell, and McLennan (1994).

Discounted stochastic games have been introduced by Shapley (1953) as a general model of strategic interaction with symmetric information and have since been intensively analyzed in both the economic and the mathematical literature. Since a full characterization of equilibria in stochastic games typically is intractable, one usually tries to prove the existence of timehomogeneous equilibria in Markovian strategies. Such equilibria are appealing as they are subgame-perfect and because, in equilibrium, the dynamics of the state sequence can be described by a homogeneous Markov chain. For finite, or, more generally, countable state spaces a variety of existence theorems have been established by, e.g., Shapley (1953), Parthasarathy (1982), and Federgruen (1978). The existence of homogeneous Markov equilibria has also been proved in special cases with uncountable state spaces, see, e.g., Parthasarathy and Sinha (1989) for a result for games with state independent transitions. However, no general existence result is yet known. Even less is known about the asymptotic behavior of the state sequence in equilibrium. In the context of finite-horizon stochastic games with mutually absolutely continuous transition probabilities Duffie et al. (1994) prove the existence of a stationary correlated equilibria. In a correlated equilibrium the behavior of the players is coordinated by a public signal (a "sunspot") transmitted by a fictitious mediator. These authors also show that the equilibrium process admits a stationary measure, that is, an initial distribution such that, in equilibrium, the state sequence is stationary and ergodic. According to Duffie et al. (1994), there are a variety of reasons to focus on such equilibria: Stationary equilibria "constitute the simplest sort of equilibria and are thus perhaps focal," and "there is (...) the suspicion that other equilibria require implausible (...) coordina-

tion." In addition, Guesnerie and Woodford (1992) argue that "an equilibrium that does not display minimal regularity through time - maybe stationarity - is unlikely to generate the coordination between agents that it assumes." 
This paper extends some of the results proved in Duffie et al. (1994) to infinite horizon stochastic games. Using a perturbation of the Moderate Social Influence condition introduced in Horst and Scheinkman (2002), we show that stochastic games with compact state and action spaces have homogeneous equilibria if the interaction between different players is not too strong. Our proof is based on the observation that discounted stochastic games with weak interactions have Nash equilibria in Markovian strategies that depend in a Lipschitz continuous manner on the current state. This part of the paper is very much inspired by the work of Curtat (1996) who analyzed supermodular stochastic games.

Supermodular games are games in which the marginal returns to increasing one's own action rise with increases in the competitors' action. Such games have recently been attracted much attention as they provide a unified approach to analyzing games with strategic complementarities; see, e.g., Milgrom and Roberts (1990), Topkis (1998) and, in particular, Amir (1996a, 1996b) for applications of supermodular games to the Cournot oligopoly and to a capital accumulation problem, respectively. However, in view of many applications like microstructure models for financial markets, it seems natural to relax the strategic complementarity condition. This calls for an extension of Curtat's result to more general stochastic games which we carry out in Section 2.1. For example, the state sequence could be a process which describes the stochastic evolution of the mood of investors who are active on a financial market. An investor's opinion on the value a risky asset typically does not only depend on his subjective perception of some underlying fundamental value, but is often also based on the recommendations of financial experts or fund managers. The behavior of these market participants typically affects the decisions of many small investors, and has thus a strong impact on the evolution of stock prices. It seems natural to assume that these big players anticipate the feedback effect their actions have on the formation of asset prices and, therefore, interact in a strategic manner. However, the players usually have conflicting interests, and so there is no reason to assume that the underlying stochastic game displays strategic complementarities.

Unlike Curtat (1996), our proof of the existence of Lipschitz continuous equilibria does not need Topki's (1978) Monotonicity Theorem. Instead of assuming strategic complementarities we consider stochastic games in which the interaction between different players is sufficiently weak and view an agents' optimization problem as an optimization problem depending on a parameter. Montrucchio (1987) shows that such problems have optimal solutions that 
are Lipschitz continuous functions of the parameter, and this allows us to prove our main result. In case the game has norm-continuous transition probabilities, the induced equilibrium process can then be described by a Markov chain which has the Feller property. The existence of stationary equilibria in discounted stochastic games with compact state spaces and weakly interacting players thus follows from standard results from the theory of Markov chains on compact state spaces.

In Section 3 we consider a special class of stochastic games which we shall refer to as Stochastic Games with Complete Connections. For such games a stronger result can be established. Assuming that the interaction between the players is not too strong we show that stochastic games with complete connections have ergodic equilibria. That is, a Nash equilibrium in Markovian strategies that does not only have an ergodic measure, but which has the additional property that the induced state sequence converges in law to a unique limiting distribution. Stochastic games with complete connections arise in certain classes of microstructure models for financial markets as we shall illustrate in Section 4.

The remainder of the paper is organized as follows. In Section 2 we state conditions on discounted stochastic games with guarantee the existence of Lipschitz continuous equilibria in Markovian strategies and prove the existence of stationary equilibria for games with compact state spaces and with weak interactions. Section 3 analyzes stochastic games with complete connections. In Section 4 we apply our game-theoretic results in order to analyze the asymptotic behavior of asset prices in a simple financial market model. Section 5 concludes.

\section{Lipschitz continuous Nash equilibria in stochastic games}

In this section we study non-zero sum non-cooperative discounted stochastic games with compact and convex action, but with possibly unbounded state spaces. We formulate conditions on the utility functions and on the law of motion governing the dynamics of the state sequence, which guarantee the existence of homogeneous Lipschitz continuous equilibria in Markov strategies. Unlike in the framework of supermodular games studied in Curtat (1996), we do not need to impose strategic complementarity or monotonicity conditions on the game. Instead, we shall work under a Moderate Social Influence condition. This means that the interaction between different players is not too strong and that the impact of an individual player on the dynamics of the state sequence is sufficiently weak. 


\subsection{The stochastic game}

Let us now be more specific about the structure of the stochastic games we are going to analyze. We define a discounted stochastic game as a tuple $\Sigma=\left(I, M,\left(\underline{\bar{X}}^{i}, U^{i}, \beta^{i}\right), Q, \xi\right)$ with the following components:

- $I=\{1,2, \ldots, N\}$ is a finite set of players.

- $M$ is a possibly unbounded normed state space equipped with its Borel- $\sigma$-field $\mathcal{M}$.

- $\underline{\bar{X}}^{i}$ is the compact and convex normed action space for player $i \in I$.

- $U^{i}: M \times \prod_{i \in I} \underline{X}^{i} \rightarrow \mathbb{R}$ is the utility functionfor player $i \in I$.

- $\beta^{i} \in(0,1)$ is the discount factor for player $i \in I$.

- $Q$ is the law of motion, i.e., a stochastic kernel from $M \times \prod_{i \in I} \underline{\bar{X}}^{i}$ to $M$ which governs the conditional transition dynamics of the state sequence.

- $\xi \in M$ is the starting point of the state sequence.

A typical action of player $i \in I$ is denoted $x^{i} \in \underline{X}^{i}$. The actions taken his competitors are denoted $x^{-i} \in \underline{\bar{X}}^{-i}:=\left\{x^{-i}=\left(x^{j}\right)_{j \in I \backslash\{i\}}\right\}$, and $\underline{\bar{X}}:=\left\{x=\left(x^{i}\right)_{i \in I}: x^{i} \in \underline{\bar{X}}^{i}\right\}$ is the compact set of all action profiles.

We consider stochastic games in which actions are chosen at discrete times $t=1,2, \ldots$ At each time $t \in \mathbb{N}$, the players observe the current position $\xi_{t} \in M$ of the state sequence. They take their actions $x_{t}^{i}=\tau^{i}\left(\xi_{t}\right)$ independently of each other according to a homogeneous Markov strategy $\tau^{i}: M \rightarrow \underline{\bar{X}}^{i}$. The selected action profile $x_{t}=\left(x_{t}^{i}\right)_{i \in I}$ along with the present state $\xi_{t}$ yields the instantaneous payoff $U^{i}\left(\xi_{t}, x_{t}\right)=U^{i}\left(\xi_{t}, x_{t}^{i}, x_{t}^{-i}\right)$ to the agent $i \in I$, and the conditional distribution of the new state $\xi_{t+1}$ in the following period $t+1$ is given by $Q\left(\xi_{t}, x_{t} ; \cdot\right)$. We denote by $\Omega:=M^{\mathbb{N}}$ the path space of the state sequence $\left\{\xi_{t}\right\}$ and by $\mathcal{F}$ the product- $\sigma$-field on $\Omega$. A homogeneous Markov strategy $\tau=\left(\tau^{i}\right)_{i \in I}$ along with an initial distribution $\mu$ for the starting point of the state sequence and together with the law of motion $Q$ induces a probability measure $\mathbb{P}_{\mu}^{\tau}$ on $(\Omega, \mathcal{F})$ in the canonical way. Under the measure $\mathbb{P}_{\mu}^{\tau}$ the state sequence $\left\{\xi_{t}\right\}$ is a Markov chain on the state space $M$. 
Remark 2.1 Recall that the transition operator $K^{\tau}$ of the Markov chain $\left(\left\{\xi_{t}\right\}, \mathbb{P}_{\delta_{\xi}}^{\tau}\right)$ acts on a bounded measurable function $f: M \rightarrow M$ according to

$$
K^{\tau} f(\cdot):=\int_{M} f(\eta) K^{\tau}(\cdot ; d \eta)=\int_{M} f(\eta) Q(\cdot, \tau(\cdot) ; d \eta)
$$

For a stationary strategy $\tau$, the expected discounted reward to player $i \in I$ is given by

$$
J^{i}(\xi, \tau)=J^{i}\left(\xi, \tau^{i}, \tau^{-i}\right):=\mathbb{E}_{\xi}^{\tau}\left[\sum_{t=0}^{\infty}\left(\beta^{i}\right)^{t} U^{i}\left(\xi_{t}, x_{t}\right)\right] .
$$

$\underset{\xi}{H e r e} \mathbb{P}_{\gamma_{\xi}} e_{\xi}$

sh equilibrium for $\Sigma$ if no players can increase

, if

$J^{i}\left(\xi, \sigma^{i}, \tau^{-i}\right)$

$\underline{\underline{X}}^{i}$ and each $i \in I$.

The existence of $(\epsilon-)$ equilibrium strategies for discounted non-zero sum stochastic games has been established under rather general assumptions on the state and the action spaces and on the players' utility functions; see, e.g., Mertens and Parthasarathy (1987), Nowak (1985) or Duffie et al. (1994). The existence of equilibria which display additional continuity properties, however, turns out to be more involved. To the best of our knowledge, this issue has so far only been addressed in the context of supermodular games by Curtat (1996), and in a game of stochastic capital accumulation by Amir (1996b). In the following section we are going to generalize this results to a larger class of discounted stochastic games where the action spaces are compact and convex subsets of Hilbert spaces. At the same time, we aim at filling what appears - at least to us - to be a gap in Curtat's theory.

\section{$2.2 \quad$ Assumptions and the main results}

For the remainder of this section we assume that the action and state spaces satisfy the following conditions.

Assumption 2.2 (i) The action space $\underline{\bar{X}}^{i}$ is a closed, compact and convex subset of some Hilbert space $\left(H^{i},\|\cdot\|_{i}\right)$.

(ii) The state space $M$ is a convex subset of a normed space $\left(H,\|\cdot\|_{M}\right)$. 
The norm on the product space $\underline{\bar{X}}$ is defined by $\|x\|^{2}:=\sum_{i \in I}\left\|x^{i}\right\|_{i}^{2}$. Before we give conditions of the stochastic game $\Sigma$ which guarantee the existence of a Lipschitz continuous Nash equilibrium, we recall the notion of an $\alpha$-concave function.

Definition 2.3 Let $Y$ be a convex subset of a Hilbert space $H$ and let $\alpha>0$ be a positive constant. Following Montrucchio (1987) we say that the function $f: Y \rightarrow \mathbb{R}$ is $\alpha$-concave if the map $y \mapsto f(y)+\frac{1}{2} \alpha\|y\|^{2}$ is concave on $Y$.

In the differentiable case, there are simple criteria to verify $\alpha$-concavity. For example, if $f$ is concave and twice differentiable on an open set $Y_{1}$ containing $Y$, then $f$ is $\alpha$-concave whenever

$$
\left|y^{t} D^{2} f\left(y_{1}\right) y\right| \geq \alpha\|y\|^{2} \quad \text { for all } y_{1} \in Y_{1} \text { and } y \in Y .
$$

A twice differentiable function $f:[a, b] \rightarrow \mathbb{R}$ is $\alpha$-concave if $f^{\prime \prime} \leq-\alpha$.

We will also need the notion of directional derivatives.

Definition 2.4 Let $Y$ be a convex subset of a Hilbert space $H$ and let $f: Y \rightarrow \mathbb{R}$ be a finite function. $f$ is differentiable at $y \in Y$ in the feasible direction $h \in H$ if $y+$ th $\in H$ for some $t>0$ and if the limit

$$
f^{\prime}(y ; h):=\lim _{t \downarrow 0} \frac{1}{t}(f(y+t h)-f(y))
$$

is exists and is finite.

In terms of directional derivatives, we have the following characterization of $\alpha$-concavity which is a corollary to Propositions 4.8 and 4.12 in Vival (1983).

Lemma 2.5 Let $Y$ be a convex subset of a Hilbert space $(H,\|\cdot\|)$. A function $f: Y \rightarrow \mathbb{R}$ is $\alpha$-concave if and only if one of the following conditions is satisfied:

$$
f\left(y_{1}\right)-f\left(y_{2}\right)-f^{\prime}\left(y_{2} ; y_{2}-y_{1}\right) \leq-\frac{1}{2} \alpha\left\|y_{2}-y_{1}\right\|^{2} \quad\left(y_{1}, y_{2} \in Y\right)
$$

or

$f$ is locally Lipschitz continuous and $f^{\prime}\left(y_{1} ; y_{1}-y_{2}\right)-f^{\prime}\left(y_{2} ; y_{1}-y_{2}\right) \leq-\alpha\left\|y_{1}-y_{2}\right\|^{2}$.

We are now going to formulate our assumptions on the utility functions $U^{i}$. We need to assume strong concavity of an agents' utility function which respect to his own action and need to place a quantitative bound on the dependence of his instantaneous utility on the actions taken by his competitors. 
Assumption 2.6 $\quad$ (i) The utility functions $U^{i}: M \times \underline{\bar{X}} \rightarrow \mathbb{R}$ are bounded and Lipschitz continuous:

$$
\left|U^{i}\left(\xi_{1}, x\right)-U^{i}\left(\xi_{2}, y\right)\right| \leq L\left(\left\|\xi_{1}-\xi_{2}\right\|_{M}+\|x-y\|\right)
$$

for some $L<\infty$ and for all $\xi_{1}, \xi_{2} \in M$ and each $x, y \in \underline{\bar{X}}$.

(ii) The map $U^{i}\left(\xi, \cdot, x^{-i}\right)$ is $\alpha(\xi)$-concave on $\underline{\bar{X}}^{i}$ for all $x^{-i} \in \underline{\bar{X}}^{-i}$ and $\inf _{\xi \in M} \alpha(\xi)>0$.

(iii) There are constants $L^{i, j}(\xi)$ such that he partial derivative $U_{1}^{i}\left(\xi, x ; h^{i}\right)$ of the utility function $U^{i}$ at $(\xi, x)$ in the direction $h^{i} \in H^{i}$ satisfies

$$
\left|U_{1}^{i}\left(\xi, x^{i}, x^{-i} ; h^{i}\right)-U_{1}^{i}\left(\xi, x^{i}, y^{-i} ; h^{i}\right)\right| \leq L^{i, j}(\xi)\left\|x^{j}-y^{j}\right\|_{j}\left\|h^{i}\right\|_{i}
$$

for all actions profiles $x^{-i}, y^{-i} \in \underline{\bar{X}}^{-i}$ with $x^{k}=y^{k}$ for $k \notin\{i, j\}$. Moreover there are constants $\hat{L}^{i}$ such that

$$
\left|U_{1}^{i}\left(\xi_{1}, x^{i}, x^{-i} ; h^{i}\right)-U_{1}^{i}\left(\xi_{2}, x^{i}, x^{-i} ; h^{i}\right)\right| \leq L^{i}\left\|\xi_{1}-\xi_{2}\right\|_{M}\left\|h^{i}\right\|_{i}
$$

or all $\xi_{1}, \xi_{2} \in M$ and each $x \in \underline{X}$.

The quantity $L^{i, j}(\xi)$ may be viewed as a measure for the dependence of agent $i$ 's instantaneous utility of the choice of player $j$ if the current state is $\xi$. By analogy, $L^{i}$ measures the dependence of his one-period utility of the current position on the state sequence. If the utility functions are sufficiently smooth, these quantities can be expressed in terms of the partial derivatives of $U^{i}$ as the following example shows.

Example 2.7 $\quad$ (i) Let us assume that both $M \subset \mathbb{R}$ and $\underline{X}^{i} \subset \mathbb{R}$ are compact and convex and that the utility functions $U^{i}$ are twice continuously differentiable. In this case the map $x^{i} \mapsto U^{i}\left(\xi, x^{i}, x^{-i}\right)$ is $\alpha(\xi)$-concave on $\underline{\bar{X}}^{i}$ whenever $\frac{\partial^{2}}{\partial\left(x^{i}\right)^{2}} U^{i}(\xi, x) \leq-\alpha(\xi)<0$. The constants $L^{i, j}(\xi)$ and $L^{i}$ can be chosen as

$$
L^{i, j}(\xi)=\sup _{x}\left|\frac{\partial^{2}}{\partial x_{i} \partial x_{j}} U^{i}(\xi, x)\right| \quad \text { and } \quad L^{i}=\sup _{\xi, x}\left|\frac{\partial^{2}}{\partial x_{i} \partial \xi} U^{i}(\xi, x)\right| .
$$

(ii) Suppose now that $M, \underline{\bar{X}}^{i} \subset \mathbb{R}^{m}$ for some $m \in \mathbb{N}$ and assume again that each $U^{i}$ is twice continuously differentiable. In this case we may choose

$$
L^{i, j}(\xi)=\sup _{m_{1}, m_{2}, x}\left|\frac{\partial^{2}}{\partial x_{m_{1}}^{i} \partial x_{m_{2}}^{j}} U^{i}(\xi, x)\right| \quad \text { and } \quad L^{i}=\sup _{m_{1}, m_{2}, \xi, x}\left|\frac{\partial^{2}}{\partial x_{m_{1}}^{i} \partial \xi_{m_{2}}} U^{i}(\xi, x)\right|,
$$

where $x^{i}=\left(x_{1}^{i}, \ldots, x_{m}^{i}\right) \in \underline{\bar{X}}^{i}$ and $\xi=\left(\xi_{1}, \ldots, \xi_{m}\right) \in M$. 
In order to prove the existence of Lipschitz continuous equilibria for the game $\Sigma$ we also need to impose smoothness conditions on the law of motion.

Assumption 2.8 (i) The probability measure $Q(\xi, x ; \cdot)$ has a density $q(\xi, x, \cdot)$ with respect to some measure $\mu$ on $(M, \mathcal{M})$ and $q^{*}:=\sup _{\xi, x} \int|q(\xi, x, \eta)| \mu(d \eta)<\infty$.

(ii) The conditional densities are Lipschitz continuous functions of the parameters $\xi$ and $x$ :

$$
\left|q\left(\xi_{1}, x, \eta\right)-q\left(\xi_{2}, y, \eta\right)\right| \leq L\left(\left\|\xi_{1}-\xi_{2}\right\|_{M}+\|x-y\|\right)
$$

for some $L<\infty$ and for all $\xi_{1}, \xi_{2} \in M$ and $x, y \in \underline{\bar{X}}$.

(iii) The directional derivative $q_{i}\left(\xi, x, \eta ; h^{i}\right)$ of the density $q$ at $(\xi, x, \eta)$ in the direction $h^{i} \in$ $H^{i}$ is dominated by some function $\varphi(\eta)$ which is integrable with respect to $\mu$, and there exists functions $\hat{L}^{i, j}: M \times \underline{\bar{X}} \times M \rightarrow \mathbb{R}$ such that

$$
\left|q_{i}\left(\xi, x^{i}, x^{-i}, \eta ; h^{i}\right)-q_{i}\left(\xi, x^{i}, y^{-i}, \eta ; h^{i}\right)\right| \leq \hat{L}^{i, j}(\xi, x, \eta)\left\|x^{j}-y^{j}\right\|_{j}\left\|h^{i}\right\|_{i}
$$

if $x^{k}=y^{k}$ for all $k \notin\{i, j\}$ and such that

$$
\left|q_{i}\left(\xi, x^{i}, x^{-i}, \eta ; h^{i}\right)-q_{i}\left(\xi, \hat{x}^{i}, x^{-i}, \eta ; h^{i}\right)\right| \leq \hat{L}^{i, i}(\xi, x, \eta)\left\|x^{i}-\hat{x}^{i}\right\|_{i}\left\|h^{i}\right\|_{i}
$$

Moreover, there are constants $\hat{L}^{i}$ such that

$$
\left|q_{i}\left(\xi_{1}, x, \eta ; h^{i}\right)-q_{i}\left(\xi_{2}, x, \eta ; h^{i}\right)\right| \leq \hat{L}^{i}\left\|\xi_{1}-\xi_{2}\right\|_{M}\left\|h^{i}\right\|_{i}
$$

for each $\xi_{1}, \xi_{2} \in M$ and all $x=\left(x^{i}, x^{-i}\right) \in \underline{\bar{X}}$.

Observe that the Lipschitz continuity condition (4) translates into a norm-continuity condition on the transition probabilities $Q(\xi, x ; \cdot)$. If $\xi_{n} \rightarrow \xi$ and $x_{n} \rightarrow x$, then

$$
\sup _{B \in \mathcal{M}}\left|Q\left(\xi_{n}, x_{n} ; B\right)-Q(\xi, x ; B)\right| \rightarrow 0 \quad \text { as } n \rightarrow \infty
$$

Such an assumption has also been imposed by, e.g., Mertens and Parthasarathy (1987) and Duffie et al. (1994). Note also that in case the conditional densities are smooth enough the random variables $\hat{L}^{i, j}(\xi, x, \eta)$ may be chosen as

$$
\hat{L}^{i, j}(\xi, x, \eta)=\frac{\partial^{2}}{\partial x_{i} \partial x_{j}} q(\xi, x, \eta)
$$


In order to guarantee the existence of Lipschitz continuous equilibria in discounted stochastic games we need to assume that the interaction between different agents is not too strong. Since the players interact through their utility functions and through their individual impacts on the evolution of the state sequence, we need to control both the impact of an action of player $j$ on the instantaneous reward to the agent $i$ and the dependence of the law of motion on the actions taken by an individual player. We formulate this conditions in terms of a perturbation of the weak interaction assumption introduced in Horst and Scheinkman (2002). To this end, we denote by

$$
\hat{L}^{i, j}(\xi):=\sup _{x}\left\|\hat{L}^{i, j}(\xi, x, \cdot)\right\|_{L^{1}}
$$

the canonical upper bound of the $L^{1}$-norms of the random variables $\hat{L}^{i, j}(\xi, x, \cdot)$ with respect to the measure $\mu$ and by $\|\cdot\|_{\infty}$ the usual sup-norm. We say that Moderate Social Influence occurs if the following condition is satisfied.

Assumption 2.9 There exists $\gamma<1$ such that

$$
\frac{\sum_{j \neq i} L^{i, j}(\xi)+\frac{\beta^{i}}{1-\beta^{i}}\left\|U^{i}\right\|_{\infty} \sum_{j \in I} \hat{L}^{i, j}(\xi)}{\alpha(\xi)} \leq \gamma .
$$

Before we state the main result of this section, we consider some case studies where our Moderate Social Influence Assumption 2.9 can indeed be verified. A detailed discussion including a motivation of Assumption 2.9 is postponed to the end of Section 2.4.

Example 2.10 Assume that $M \subset \mathbb{R}$ and that $\underline{\bar{X}}^{i}=[0,1]$. Assume moreover that the players' utility functions $U^{i}$ are twice continuously differentiable on an open set containing $\underline{\bar{X}}$. Consider a law of motion $Q$ which is of the form

$$
Q(\xi, x ; \cdot)=\frac{\sum_{i \in I} x^{i}}{N} Q_{1}(\xi ; \cdot)+\left(1-\frac{\sum_{i \in I} x^{i}}{N}\right) Q_{1}(\xi ; \cdot) .
$$

If the measure $Q_{i}(\xi ; \cdot)$ has a density $q_{i}(\xi, \cdot)$ with respect to some law $\mu$ on $\mathbb{R}$ that satisfies

$$
\left|q_{i}\left(\xi_{1}, \eta\right)-q_{i}\left(\xi_{2}, \eta\right)\right| \leq L\left|\xi_{1}-\xi_{2}\right| \quad(i=1,2)
$$

then our Moderate Social Influence condition translates into an assumption on the marginal rates of substitution. Indeed, in our present setting we may choose $\hat{L}^{i, j}(\xi) \equiv 0$, and so ( 7$)$ holds if, for example,

$$
\sum_{j \neq i}\left|\frac{\partial^{2}}{\partial x_{i} \partial x_{j}} U^{i}(\xi, x)\right| \leq \gamma\left|\frac{\partial^{2}}{\partial\left(x_{i}\right)^{2}} U^{i}(\xi, x)\right|
$$

for all $i \in I$ and for some $\gamma<1$. 
Let us now show how the dynamic extension of Diamond's (1982) search model studied in Curtat (1996) fits into our framework.

Example 2.11 Consider Curtat's (1996) dynamic extension of the Diamond search model. In this case $\underline{\bar{X}}^{i}=[0,1]$ and $M=[1,2]$. The one-period reward functions are given by

$$
U^{i}(\xi, x)=\xi x^{i} \sum_{j \neq i} x^{j}-1.74\left(1+x^{i}\right)^{3}
$$

and the law of motion is of the form

$$
Q(\xi, x ; \cdot)=\frac{\xi+x^{1}+\cdots+x^{N}}{2+N} Q_{1}(\cdot)+\left(1-\frac{\xi+x^{1}+\cdots+x^{N}}{2+N}\right) Q_{2}(\cdot) .
$$

In view of Example 2.10, the game has a Lipschitz continuous equilibrium if $N \leq 5$ because

$$
\frac{\partial^{2}}{\partial x^{i} \partial x^{j}} U^{i}(\xi, x)=\xi \leq 2 \quad \text { and } \quad \frac{\partial^{2}}{\partial\left(x^{i}\right)^{2}} U^{i}(\xi, x)=-10.44\left(1+x^{i}\right) \leq-10.44 .
$$

Among others, Nowak (1987) and Küenle (1999) consider stochastic games with additive reward and additive transition structure. For such games our Moderate Social Influence conditions also takes a particularly simple form.

Example 2.12 Consider an ARAT game, i.e., a stochastic game where both the reward functions and the transition probabilities are the sum of $N$ terms, and where the $i$-th term depends only on the action taken by the player $i \in I$. More precisely, assume that

$$
U^{i}(\xi, x)=\sum_{j \in I} u^{i, j}\left(\xi, x^{j}\right) \quad \text { and that } \quad Q(\xi, x ; \cdot)=\sum_{j \in I} Q^{i}\left(\xi, x^{i} ; \cdot\right)
$$

Assume also that the law $Q^{i}\left(\xi, x^{i} ; \cdot\right)$ has a Lipschitz continuous and sufficiently smooth density $q^{i}\left(\xi, x^{i} ; \cdot\right)$ with respect to some measure $\mu^{i}$ and that the map $x^{i} \mapsto u^{i, i}\left(\xi, x^{i}\right)$ is $\alpha(\xi)$-concave on $\underline{\bar{X}}^{i}$. In such a situation moderate social interaction prevails if there is $\gamma<1$ such that

$$
\max _{i \in I}\left\{\frac{\beta^{i}}{1-\beta^{i}}\left\|U^{i}\right\|_{\infty} \sup _{x}\left\|\frac{\partial^{2}}{\partial\left(x^{i}\right)^{2}} q^{i}\left(\xi, x^{i} ; \cdot\right)\right\|_{L^{1}}\right\} \leq \gamma \alpha(\xi) .
$$

We are now going to state a first result which guarantees the existence of Lipschitz continuous equilibria in stochastic games with compact state spaces. It will be proved in Section 2.5 below. 
Theorem 2.13 Every discounted stochastic game $\Sigma$ with a compact state space that satisfies Assumptions 2.2, 2.6, 2.8 and 2.9 has a homogeneous equilibrium in Markov strategies $\tau$ which is Lipschitz continuous. That is, there exists $L^{*}<\infty$ such that

$$
\left\|\tau^{i}\left(\xi_{1}\right)-\tau^{i}\left(\xi_{2}\right)\right\|_{i} \leq L^{*}\left\|\xi_{1}-\xi_{2}\right\|_{M} \quad(i \in I)
$$

Theorem 2.13 may also be viewed as an extension of Theorem 4.1 in Duffie et al. (1994). In the context of finite-horizon stochastic games and under a mutual absolute continuity assumption on the conditional transition laws - but without any kind of Moderate Social Influence assumption - these authors obtain the existence of a "special kind of (stationary) correlated equilibrium" for discounted stochastic games. That is, they obtain the existence of a correlated equilibrium where the equilibrium process admits an ergodic measure.

Definition 2.14 Following Duffie et al. (1994) we say that a discounted stochastic game $\Sigma$ has a stationary equilibrium if there exists a homogeneous equilibrium in Markovian strategies $\tau$ and an initial distribution $\mu$ on $(M, \mathcal{M})$ such that the Markov chain $\left(\left\{\xi_{t}\right\}, \mathbb{P}_{\mu}^{\tau}\right)$ is stationary and ergodic.

The following theorem shows that a discounted stochastic game with compact state space has a stationary equilibrium, whenever the interaction between different agents is not too strong.

Theorem 2.15 Every discounted stochastic game $\Sigma$ which satisfies the assumptions of Theorem 2.13 admits a stationary equilibrium.

Theorem 2.15 guarantees the existence of a Markov strategy $\tau$ and an initial distribution $\mu$ such that the equilibrium process is stationary and ergodic under $\mathbb{P}_{\mu}^{\tau}$. An extension of this result will be established in Section 3 where we introduce a new class of stochastic games called Stochastic Games with Complete Connections. We shall see that such games admit a homogeneous Markov strategy $\tau$ such that, independently of the initial distribution, the state sequence converges in law to a unique stationary measure.

Our Theorem 2.13 is applicable to stochastic games with compact, and hence bounded state spaces. However, in many applications it seems natural to assume an unbounded state space. This calls for an extension Theorem 2.13 to stochastic games with unbounded state spaces. Such an extension can indeed be established under a mild additional assumption on 
the conditional densities $q(\xi, x, \cdot)$. To make this more precise, let $M_{n} \uparrow M$ be an increasing sequence of compact and convex subsets of $H$ and $q_{n}(\xi, x, \cdot)(n \in \mathbb{N})$ be a sequence of conditional densities which converges to $q(\xi, x, \cdot)$ uniformly on compact sets:

$$
\sup _{\eta \in K}\left|q_{n}(\xi, x, \eta)-q(\xi, x, \eta)\right| \stackrel{n \rightarrow \infty}{\longrightarrow} 0 \quad \text { for all compact sets } K \subset M .
$$

Remark 2.16 Let $Q_{n}$ be the stochastic kernel from $M_{n} \times \underline{\bar{X}}$ to $M_{n}$ that is defined in terms of the conditional density $q_{n}$ and consider the stochastic game $\Sigma_{n}=\left(I, M_{n},\left(U^{i}, \underline{\bar{X}}^{i}, \beta^{i}\right), Q_{n}, \xi\right)$. Our condition (8) translates into an assumption on the conditional transition dynamics of the state sequences $\left\{\xi_{t}^{n}\right\}$ and $\left\{\xi_{t}\right\}$ associated to the respective games $\Sigma_{n}$ and $\Sigma$. In order to see this, we fix a state $\xi \in M_{n}$ and an action profile $x \in \underline{\bar{X}}$ and introduce the measures $\mu_{n}(\xi, x ; \cdot)$ and $\mu(\xi, x ; \cdot)$ by

$$
d \mu_{n}(\xi, x ; \cdot)=q_{n}(\xi, x ; \cdot) d \mu \quad \text { and } \quad d \mu(\xi, x ; \cdot)=q(\xi, x ; \cdot) d \mu
$$

respectively. For any bounded function $h: H \rightarrow \mathbb{R}$ with compact support $K \subset H$ we have

$$
\lim _{n \rightarrow \infty}\left|\int_{K} h(\eta)\left[\mu_{n}(\xi, x ; d \eta)-\mu(\xi, x ; d \eta)\right]\right| \leq\|h\|_{\infty} \sup _{\eta \in K}\left|q_{n}(\xi, x, \eta)-q(\xi, x, \eta)\right|=0 .
$$

Thus, under (8) the sequence of conditional transition probabilities $\left\{\mu_{n}(\xi, x ; \cdot)\right\}$ converges weakly to the probability measure $\mu(\xi, x ; \cdot)$.

We are now ready to formulate an extension of Theorem 2.13 to stochastic games with unbounded state spaces which will be proved below.

Theorem 2.17 Let $\Sigma=\left(I, M,\left(\underline{\bar{X}}^{i}, U^{i}, \beta^{i}\right), Q, \xi\right)$ be a discounted non-cooperative stochastic game that satisfies the following conditions:

(i) The action spaces satisfy Assumption 2.2 (i).

(ii) The utility functions satisfy Assumption 2.6.

(iii) The law of motion $Q$ satisfies Assumption 2.8.

(iv) There exists an increasing sequence of closed compact convex sets $M_{n} \uparrow M$ and a family of conditional densities $\left(q_{n}\right)$ satisfying (8) and Assumption 2.8 (ii) - (iv).

Then the game $\Sigma$ has a Lipschitz continuous stationary Nash equilibrium in Markov strategies. 
Let us illustrate our Theorem 2.17 in a situation where the interaction between different players is local on the level of instantaneous utilities and global on the level of the law of motion.

Example 2.18 Let $M=\mathbb{R}$ and $\underline{\bar{X}}^{i}=[-1,1]$. Let $\varphi(m, \cdot)$ be the density of the normal distribution with mean $m$ and standard deviation 1. Assume that $Q(\xi, x ; \cdot)=q(\xi, x, \eta) d \eta$ and that

$$
q(\xi, x ; \cdot)=\varphi\left(\xi+\frac{\sum_{i=1}^{N} x^{i}}{N}, \cdot\right)
$$

Thus, for games with many players, the impact of an individual agent on the distribution of the new state is weak. It is easily seen that these densities satisfy (8). Moreover, a straightforward calculation shows that

$$
\hat{L}^{i, j}(\xi) \leq \sup _{x}\left\|\frac{\partial^{2}}{\partial x^{i} \partial x^{j}} q(\xi, x ; \cdot)\right\|_{L^{1}} \leq \frac{c}{N^{2}} \quad \text { for some } c<\infty \text { and for all } i, j \in I .
$$

Let us also assume that the direct interaction between different players is local in the sense that $U^{i}(\xi, x)$ depends on the entire action profile $x \in \underline{\bar{X}}$ only through $x^{i}$ and through the action $x^{j}$ taken by player $i$ 's neighbor, agent $j=(i+1) \bmod N$. In this case $L^{i, j}(\cdot) \equiv 0$ for all $j \neq(i+1) \bmod N$ and moderate social influence occurs if

$$
\left|\frac{\partial^{2}}{\partial x^{i} \partial x^{i+1}} U^{i}(\xi, x)\right| \leq \gamma\left|\frac{\partial^{2}}{\partial\left(x^{i}\right)^{2}} U^{i}(\xi, x)\right|
$$

for some $\gamma<1$ and if $N$ is sufficiently large.

\subsection{Lipschitz continuous solutions to parameterized optimization problems}

Unlike Curtat (1996), our existence proof for Lipschitz continuous Nash equilibria in discounted stochastic games does no need Topki's (1978) Monotonicity Theorem. Instead, we combine our Moderate Social Influence condition with a result in Montrucchio (1987). Montrucchio gives general conditions under which a collection of optimization problems, with the objective function depending on a parameter, has optimal solutions that are Lipschitz continuous functions of the parameter. More precisely, in his Theorem 3.1 he proves the following:

Theorem 2.19 Let $X$ be a closed and convex subset of some Hilbert space $\left(H_{1},\|\cdot\|_{1}\right)$ and let $Y$ be a convex subset of a normed space $\left(H_{2},\|\cdot\|_{2}\right)$. Let $F: X \times Y \rightarrow \mathbb{R}$ be a finite function which satisfies the following conditions: ${ }^{1}$ 
(i) For all $y \in Y$, the map $x \mapsto F(x, y)$ is $\alpha$-concave and upper-semicontinuous on $X$.

(ii) For all feasible $h \in H$, the directional derivative $F_{1}(x, y ; h)$ of $F$ at $(x, y)$ in the direction $h$ satisfies the Lipschitz continuity condition

$$
\left|F_{1}\left(x, y_{1} ; h\right)-F_{1}\left(x, y_{2} ; h\right)\right| \leq L\left\|y_{1}-y_{2}\right\|_{2}\|h\|_{1}
$$

for all $y_{1}, y_{2} \in Y$ and all $x \in X$.

Under the above assumptions there exists a unique map $\theta: Y \rightarrow X$ that satisfies $\sup _{x \in X}=$ $F(x, y)=F(\theta(y), y)$. Moreover, $\theta$ is Lipschitz continuous and

$$
\left|\theta\left(y_{1}\right)-\theta\left(y_{2}\right)\right| \leq \frac{L}{\alpha}\left\|y_{1}-y_{2}\right\|_{2}
$$

for all $y_{1}, y_{2} \in Y$.

\subsection{Lipschitz continuous equilibria in a static one-shot game}

Let us prepare the proof of Theorem 2.13. To this end, we introduce the vector $u=\left(u^{i}\right)_{i \in I}$ with components $u_{i}:=\left\|U^{i}\right\|_{\infty}$ and denote by $\left(B_{u}\left(M, \mathbb{R}^{N}\right),\|\cdot\|_{\infty}\right)$ the Banach space of all measurable functions $f: M \rightarrow \mathbb{R}^{N}$ satisfying $\left\|f^{i}\right\|_{\infty} \leq u^{i}$. To each such average continuation function we associate the reduced one-shot game $\Sigma_{f}:=\left(I, M,\left(\underline{\bar{X}}^{i}, U^{i, f}\right), \xi\right)$ with payoff functions

$$
U^{i, f}(\xi, x)=\left(1-\beta^{i}\right) U^{i}(\xi, x)+\beta^{i} \int_{M} f^{i}(\eta) q(\xi, x, \eta) \mu(d \eta)
$$

Remark 2.20 Notice that $U^{i, f}(\xi, x)$ is the payoff to player $i \in I$ in the stochastic game $\Sigma$, if the game terminates after the first round, if the players receive rewards according to the payoff functions $f^{i}$ in the second period, and if first period payoffs are discounted at the respective rates $1-\beta^{i}$.

Our aim is now threefold. We wish to sow that (i) the conditional best reply $g_{f}^{i}\left(\xi, x^{-i}\right)$ of the player $i \in I$, given the actions $x^{-i} \in \underline{\bar{X}}^{-i}$ of his competitors, is uniquely determined, (ii) the reduced game $\Sigma_{f}$ has a unique equilibrium $g_{f}(\xi)$ and (iii) the map $g_{f}: M \rightarrow \underline{\bar{X}}$ is Lipschitz continuous with a constant that is independent of the average continuation function. The latter property turns out to be the key to the proof of Theorem 2.13.

\footnotetext{
${ }^{1}$ Montrucchio (1987) formulated this theorem under the additional assumption of $Y$ being a closed and convex subset of a Hilbert space $\mathrm{H}_{2}$. His proof, however, shows that this assumption is redundant.
} 
Lemma 2.21 Under the Assumptions of Theorem 2.13 the following holds:

(i) For each $\xi \in M$ and $x^{-i} \in \underline{\bar{X}}^{-i}$, the map $x^{i} \mapsto U^{i, f}\left(\xi, x^{i}, x^{-i}\right)$ is $\hat{\alpha}^{i}(\xi)$-concave on $\underline{\bar{X}}^{i}$. The concavity parameter $\hat{\alpha}^{i}(\xi)$ if given by

$$
\hat{\alpha}^{i}(\xi)=\min _{i}\left\{\left(1-\beta^{i}\right) \alpha(\xi)-u^{i} \beta^{i} \hat{L}^{i, i}(\xi)\right\}
$$

and $\inf _{\xi} \hat{\alpha}^{i}(\xi)>0$.

(ii) The conditional best rely $g_{f}^{i}\left(\xi, x^{-i}\right)$ of player $i \in I$ depends in a Lipschitz continuous manner on the actions of his competitors. More precisely, we have

$$
\left\|g_{f}^{i}\left(\xi, x^{-i}\right)-g_{f}^{i}\left(\xi, y^{-i}\right)\right\|_{i} \leq \frac{\left(1-\beta^{i}\right) L^{i, j}(\xi)+u^{i} \beta^{i} \hat{L}^{i, j}(\xi)}{\hat{\alpha}^{i}(\xi)}\left\|x^{j}-y^{j}\right\|_{j}
$$

if $x^{k}=y^{k}$ for all $k \neq j$. Moreover, there exists $\tilde{L}<\infty$ such that

$$
\left\|g_{f}^{i}\left(\xi_{1}, x^{-i}\right)-g_{f}^{i}\left(\xi_{2}, x^{-i}\right)\right\|_{i} \leq \tilde{L}\left\|\xi_{1}-\xi_{2}\right\|_{M}
$$

for all $\xi_{1}, \xi_{2} \in M$ and each $x^{-i} \in \underline{\bar{X}}^{-i}$.

(iii) The reduced game $\Sigma_{f}$ has a unique equilibrium $g_{f}(\xi)=\left\{g_{f}^{i}(\xi)\right\}_{i \in I} \in \underline{\bar{X}}$.

(iv) The mapping $\xi \mapsto g_{f}^{i}(\xi)$ is Lipschitz continuous and the Lipschitz constant can be chosen independently of the average continuation function $f \in B_{u}\left(M, \mathbb{R}^{N}\right)$.

(v) The map $f \mapsto g_{f}^{i}(\cdot)$ from $B_{u}\left(M, \mathbb{R}_{+}^{N}\right)$ to $B(M, \underline{\bar{X}})$ is continuous.

\section{PROOF:}

(i) Let us fix an average continuation function $f$, an action profile $x^{-i} \in \underline{X}^{-i}$ and a state $\xi \in M$. In view of Lemma 2.5 it is enough to show that

$$
U_{1}^{i, f}\left(\xi, x^{i}, x^{-i} ; x^{i}-\hat{x}^{i}\right)-U_{1}^{i, f}\left(\xi, \hat{x}^{i}, x^{-i} ; x^{i}-\hat{x}^{i}\right) \leq-\hat{\alpha}(\xi)\left\|x^{i}-\hat{x}^{i}\right\|_{i}^{2}
$$

for all $x^{i}, \hat{x}^{i} \in \underline{\underline{X}}^{i}$. In order to prove (13), we put

$$
F^{i}\left(\xi, x^{i}, x^{-i}\right):=\int_{M} f^{i}(\eta) q\left(\xi, x^{i}, x^{-i}, \eta\right) \mu(d \eta)
$$

By Assumption 2.8 (iii), the directional derivative $F_{1}^{i}\left(\xi, x^{i}, x^{-i} ; x^{i}-\hat{x}^{i}\right)$ of the map $x^{i} \mapsto F^{i}\left(\xi, x^{i}, x^{-i}\right)$ at $(\xi, x)$ in the direction $x^{i}-\hat{x}^{i}$ exists and satisfies

$$
\left|F_{1}^{i}\left(\xi, x^{i}, x^{-i} ; x^{i}-\hat{x}^{i}\right)-F_{1}^{i}\left(\xi, \hat{x}^{i}, x^{-i} ; x^{i}-\hat{x}^{i}\right)\right| \leq u^{i} \hat{L}^{i, i}(\xi)\left\|x^{i}-\hat{x}^{i}\right\|_{i}^{2} .
$$


Since $U^{i}\left(\xi, \cdot, x^{-i}\right)$ is $\alpha(\xi)$-concave on $\underline{\bar{X}}^{i}$ we have

$$
U_{1}^{i}\left(\xi, x^{i}, x^{-i} ; x^{i}-\hat{x}^{i}\right)-U_{1}^{i}\left(\xi, \hat{x}^{i} ; x^{-i} ; x^{i}-\hat{x}^{i}\right) \leq-\alpha(\xi)\left\|x^{i}-\hat{x}^{i}\right\|_{i}^{2}
$$

and so (13) holds if $\left(1-\beta^{i}\right) \alpha(\xi)>\beta^{i} u^{i} \hat{L}^{i, i}(\xi)$. This, however, as well as $\inf _{\xi \in M} \hat{\alpha}^{i}(\xi)>0$ follows from the Moderate Social Influence condition.

(ii) Since an agent's utility function is strongly concave with respect to his own action, his conditional best reply given the choices of his competitors is uniquely determined. To establish the quantitative bound (11) on the dependence of player $i$ 's best reply on the behavior of all the other agents, we fix a player $j \neq i$ and action profiles $x^{-i}$ and $y^{-i}$ which differ only at the $j$-th coordinate. Under the assumptions of Theorem 2.13 the directional derivative $U_{1}^{i, f}\left(\xi, x^{i}, x^{-i} ; h^{i}\right)$ of the map $x^{i} \mapsto U^{i, f}\left(\xi, x^{i}, x^{-i}\right)$ at $(\xi, x)$ in the direction $h^{i} \in H^{i}$ satisfies

$$
\begin{aligned}
& \left|U_{1}^{i, f}\left(\xi, x^{i}, x^{-i} ; h^{i}\right)-U_{1}^{i, f}\left(\xi, x^{i}, y^{-i} ; h^{i}\right)\right| \\
\leq & \left\{\left(1-\beta^{i}\right) L^{i, j}(\xi)+\beta^{i} u^{i} \hat{L}^{i, j}(\xi)\right\}\left\|x^{j}-y^{j}\right\|_{j}\left\|h^{i}\right\|_{i} .
\end{aligned}
$$

Thus, (11) follows from Theorem 2.19. Our estimate (12) follows from similar considerations.

(iii) The existence of an equilibrium in pure strategies for the static game $\Sigma_{f}$ follows from strict concavity of the utility functions $U^{i, f}$ with respect to the player's own actions along with compactness of the action spaces using standard fixed points arguments. In order to prove uniqueness, we proceed as in the proof of Proposition 4.21 in Horst and Scheinkman (2002). Our Moderate Social Influence condition yields

$$
\hat{L}:=\sup _{i, \xi} \sum_{j \neq i} \frac{\left(1-\beta^{i}\right) L^{i, j}(\xi)+\beta^{i} u^{i} \hat{L}^{i, j}(\xi)}{\hat{\alpha}^{i}(\xi)}<1 .
$$

Thus, given the action profiles $x^{-i}$ and $y^{-i}$ of player $i$ 's competitors, it follows from (11) that

$$
\left\|g_{f}^{i}\left(\xi, x^{-i}\right)-g_{f}^{i}\left(\xi, y^{-i}\right)\right\|_{i} \leq \hat{L} \max _{j}\left\|x^{j}-y^{j}\right\|_{j}
$$

For $x \neq y$, we therefore obtain

$$
\max _{i}\left\|g_{f}^{i}\left(\xi, x^{-i}\right)-g_{f}^{i}\left(\xi, y^{-i}\right)\right\|_{i}<\max _{i}\left\|x^{i}-y^{i}\right\|_{i}
$$


Thus, the map $x \mapsto\left(g_{f}^{i}\left(\xi, x^{-i}\right)\right)$ has at most one fixed point. This proves uniqueness of equilibria in $\Sigma_{f}$.

(iv) Let $g^{f}(\xi)$ be an equilibrium. Then $g_{f}^{i}(\xi)=g_{f}^{i}\left(\xi,\left\{g_{f}^{j}(\xi)\right\}_{j \neq i}\right)$, and so

$$
\begin{aligned}
\left\|g_{f}^{i}\left(\xi_{1}\right)-g_{f}^{i}\left(\xi_{2}\right)\right\|_{i} \leq & \left\|g_{f}^{i}\left(\xi_{1},\left\{g_{f}^{j}\left(\xi_{1}\right)\right\}_{j \neq i}\right)-g_{f}^{i}\left(\xi_{1},\left\{g_{f}^{j}\left(\xi_{2}\right)\right\}_{j \neq i}\right)\right\|_{i} \\
& +\left\|g_{f}^{i}\left(\xi_{1},\left\{g_{f}^{j}\left(\xi_{2}\right)\right\}_{j \neq i}\right)-g_{f}^{i}\left(\xi_{2},\left\{g_{f}^{j}\left(\xi_{2}\right)\right\}_{j \neq i}\right)\right\|_{i} \\
\leq & \hat{L} \sum_{j \neq i}\left\|g_{f}^{j}\left(\xi_{1}\right)-g_{f}^{j}\left(\xi_{2}\right)\right\|_{j}+\tilde{L}\left\|\xi_{1}-\xi_{2}\right\|_{M} .
\end{aligned}
$$

This yields

$$
\left\|g_{f}^{i}\left(\xi_{1}\right)-g_{f}^{i}\left(\xi_{2}\right)\right\|_{i} \leq \frac{\tilde{L}}{(1-\hat{L})}\left\|\xi_{1}-\xi_{2}\right\|_{M}
$$

and so the equilibrium mapping $g_{f}: M \rightarrow \underline{\bar{X}}$ is Lipschitz continuous which a constant that does not depend on the average continuation function $f$.

(v) In order to prove this assertion we fix $\xi \in M$ and $x^{-i} \in \underline{\bar{X}}^{-i}$ and apply Theorem 2.19 to the map

$$
\left(x^{i}, f\right) \mapsto U^{i, f}\left(\xi, x^{i}, x^{-i}\right)
$$

Due to Assumption 2.8 (i) we have for all $f, g \in B_{u}\left(M, \mathbb{R}^{N}\right)$ that

$$
\left|U_{1}^{i, f_{1}}\left(\xi, x^{i}, x^{-i} ; h^{i}\right)-U_{1}^{i, f_{2}}\left(\xi, x^{i}, x^{-i} ; h^{i}\right)\right| \leq \beta^{i} q^{*}\left\|f_{1}-f_{2}\right\|_{\infty}\left\|h^{i}\right\|_{i}
$$

and so Theorem 2.19 shows that

$$
\left\|g_{f_{1}}^{i}\left(\xi, x^{-i}\right)-g_{f_{2}}^{i}\left(\xi, x^{-i}\right)\right\|_{i} \leq \frac{\beta^{i} q^{*}}{\inf _{\xi} \hat{\alpha}^{i}(\xi)}\left\|f_{1}-f_{2}\right\|_{\infty}
$$

Thus, similar arguments as in the proof of (iii) yield the assertion.

Our Moderate Social Influence conditions appears to be rather strong. However, for the proof of Theorem 2.13 it will be essential that the equilibrium mappings $g_{f}: M \rightarrow \underline{X}$ are Lipschitz continuous with a constant that does not depend on the average continuation function $f$. For this, we need uniqueness of equilibria in the reduced one-shot games $\Sigma_{f}$. We guarantee uniqueness by assuming that the utility functions $U^{i, f}$ inherit enough concavity in the player's own actions from the original reward functions $U^{i}$. This is accomplished by assuming that the dependence of an agents' utility function on the actions taken by his 
competitors is not too strong, and that the impact on an individual player on the dynamics of the state sequence is sufficiently weak. Examples 2.10 and 2.18 show that this is indeed true for games where the law of motion $Q(\xi, x ; \cdot)$ depends in a linear manner of the action chosen by the agents $i \in I$ and in games with many players where the distribution of the new state only depends on the average action and if the direct interaction between different players is local.

Remark 2.22 Lemma 2.21 may be viewed as an extension of Theorem 4.2 in Curtat (1996) to stochastic games with more general state and action spaces. However, at least to us, Curtat's result appears to be somewhat incomplete. Basically, this author assumes that $\underline{X}^{i}$ and $M$ are compact intervals, and that the transition law $Q(\xi, x ; \cdot)$ has "doubly stochastically increasing differences in $x$ and $\xi$ ". Inter alias this means that, for any increasing function $f: M \rightarrow \mathbb{R}$, the map

$$
x \hookrightarrow f(\eta) q(\xi, x, \eta) \nu(d \eta)
$$

has doubly increasing differences in $x$ and $\xi$. Thus, there is a Lipschitz continuous function $\phi: \mathbb{R} \rightarrow \mathbb{R}$ such that the map

$$
\mathscr{x} \int f(\eta) q(\xi, \phi(\xi) \mathbf{1}-x ; d \eta) \nu(d \eta)
$$

has increasing differences in $x$ and $\xi$. Here 1 denotes the vector $(1,1, \cdots, 1)$ in $\mathbb{R}^{N}$. For the proof of Theorem 4.2 in Curtat (1996) it is now essential that the Lipschitz continuous "change of variables" $\phi$ can be chosen independently of $f$. Unfortunately, the author does not give general conditions which guarantee this. In his Theorem 2.4, Curtat (1996) essentially shows that a sufficiently smooth function $F: \quad \underline{X} \times \mathbb{R} \rightarrow \mathbb{R}$ has doubly increasing differences, if and only if it has increasing differences in $x$ and $\xi$ and if it satisfies the diagonal dominance condition

$$
f_{x}^{i}:=\frac{\partial^{2} F}{\partial^{2} x^{i}}+\sum_{j \neq i} \frac{\partial^{2} F}{\partial x^{i} \partial x^{j}} \leq 0 .
$$

Applied to the mapping defined in (14), such a diagonal dominance condition holds if the conditional densities depend linearly on the players' actions, and in this case Curtat's conditions are equivalent to our Moderate Social Influence assumption. But does it also hold in more general situations? If $f_{x}^{i}<0$, then the change of variables $\phi: \mathbb{R} \rightarrow \mathbb{R}$ can be chosen as

$$
\phi(\xi)=c \xi \quad \text { where } \quad c \geq \frac{\max _{i}\left\|\frac{\partial^{2} F}{\partial x_{i} \partial \xi}\right\|_{\infty}}{\max _{i}\left\|f_{x}^{i}\right\|_{\infty}} .
$$


For the map defined by (14), this yields a change of variables whose Lipschitz constant depends on $f$. We are unaware of any conditions which ensure the existence of a change of variables which is Lipschitz continuous uniformly in the average continuation function. This motivated our Moderate Social Influence condition.

Remark 2.23 In the 2-Person stochastic capital accumulation game with convex transition probabilities studied by Amir (1996b), the conditional densities take the form $q\left(\xi-y^{1}-y^{2}, \cdot\right)$. In this special case, the Lipschitz continuous change a variables can easily be given: $\phi(\xi)=\xi$.

\subsection{Existence of Lipschitz continuous equilibria in stochastic games with bounded state spaces}

This section proves our Theorem 2.13. We therefore assume that $M \subset H$ is compact. For the average continuation function $f \in B_{u}\left(M, \mathbb{R}^{N}\right)$, we denote by $g_{f}(\xi)$ the unique equilibrium in the one-shot game $\Sigma_{f}$, and introduce an operator $T$ on $B_{u}\left(M, \mathbb{R}^{N}\right)$ by

$$
(T f)^{i}(\xi)=\left(1-\beta^{i}\right) U^{i}\left(\xi, g_{f}(\xi)\right)+\beta^{i} \int_{M} f^{i}(\eta) q\left(\xi, g_{f}(\xi), \eta\right) \mu(d \eta)
$$

A standard argument in discounted dynamic programming shows that for any fixed point $F$ of $T$, the action profile $g_{F}(\xi)$ is an equilibrium in the non-zero sum stochastic game $\Sigma$. The

equilibrium payoff to player $i \in I$ is given by $\frac{F^{i}(\xi)}{1-\beta^{i}}$, and the map $g_{F}: M \rightarrow \underline{\bar{X}}$ is Lipschitz continuous, due to Lemma 2.21.

In order to prove Theorem 2.13 it is therefore enough to establish the existence of a fixed point for the operator $T$. To this end, we will need the following basic properties of $T$.

Lemma 2.24 Under the assumptions of Theorem 2.13 the following holds:

(i) For all $f \in B_{u}\left(M, \mathbb{R}^{N}\right)$, the mapping $\xi \mapsto(T f)(\xi)$ is Lipschitz continuous.

(ii) The operator $T$ is continuous in the sense that $\lim _{n \rightarrow \infty}\left\|T f-T f_{n}\right\|_{\infty}=0$ whenever $\lim _{n \rightarrow \infty}\left\|f-f_{n}\right\|_{\infty}=0$.

PROOF:

(i) It follows from Lipschitz continuity of the utility functions and the densities that

$$
\left|(T f)^{i}\left(\xi_{1}\right)-(T f)^{i}\left(\xi_{2}\right)\right| \leq\left[\left(1-\beta^{i}\right) L+\beta^{i} L u^{i}\right]\left(\left\|\xi_{1}-\xi_{2}\right\|_{M}+\left\|g_{f}\left(\xi_{1}\right)-g_{f}\left(\xi_{2}\right)\right\|_{M}\right) .
$$


Thus, Lipschitz continuity of the mapping $\xi \mapsto g_{f}(\xi)$ yields Lipschitz continuity of $(T f)^{i}$.

(ii) To prove continuity of $T$ in the topology of uniform convergence, we fix functions $f_{n} \in B_{u}\left(M, \mathbb{R}^{N}\right)$ that converge to $f$ in the topology of uniform convergence. Lemma 2.21 (v) yields $\lim _{n \rightarrow \infty}\left\|g_{f_{n}}-g_{f}\right\|_{\infty}=0$. Thus, Lipschitz continuity of the reward functions and the densities gives us

$$
\left|\left(T f_{n}\right)^{i}(\xi)-(T f)^{i}(\xi)\right| \leq\left(1-\beta^{i}\right) L\left\|g_{f_{n}}-g_{f}\right\|_{\infty}+\beta^{i}\left\{\left\|f_{n}^{i}-f^{i}\right\|_{\infty}+u^{i} L\left\|g_{f_{n}}-g_{f}\right\|_{\infty}\right\}
$$

and so

$$
\lim _{n \rightarrow \infty}\left\|T f_{n}-T f\right\|_{\infty}=0
$$

This finishes the proof.

Remark 2.25 Our Lemma 2.24 might be viewed as a modification of Theorem 4.4 in Curtat (1996). Since the Nash equilibrium correspondence is closed valued, $\left\|f_{n}-f\right\|_{\infty} \rightarrow 0$ yields

$$
\lim _{n \rightarrow \infty} g_{f_{n}}(\xi)=g_{f}(\xi) \quad \text { for all } \xi \in M
$$

However, there is a-priori no reason to assume that the convergence is uniform over $\xi \in M$. It therefore remains unclear to us, why the operator $T$ defined in Section 4.2 in Curtat (1996) maps a certain set of increasing functions continuously, i.e., with respect to the topology of uniform convergence, into itself. From our point of view, continuity of $T$ in operator norm requires continuity of the maps $f \mapsto g_{f}(\xi)$ uniformly in $\xi \in M$.

Let $L_{g}$ be the common Lipschitz constant of the maps $g_{f}: M \rightarrow \underline{\bar{X}}$ and define

$$
L^{*}:=\max \left\{\left[\left(1-\beta^{i}\right) L+\beta^{i} L u^{i}\right]\left(1+L_{g}\right): i \in I\right\}
$$

We introduce the class $\mathcal{L}\left(L^{*}, u\right)$ of all functions $f \in B_{u}\left(M, \mathbb{R}^{N}\right)$ which are Lipschitz continuous with constant $L^{*}$. For $f \in \mathcal{L}\left(L^{*}, u\right)$ we obtain from Lemma 2.24 (i) that

$$
\left|T f_{i}\left(\xi_{1}\right)-T f_{i}\left(\xi_{2}\right)\right| \leq L^{*}\left\|\xi_{1}-\xi_{2}\right\|_{M}
$$

Thus, $T$ maps the set $\mathcal{L}\left(L^{*}, u\right)$ continuously into itself. We are now ready to prove the main results of this section. 
Proof of Theorem 2.13: Due to the Arzela-Ascoli theorem, the set $\mathcal{L}\left(L^{*}, u\right)$ is compact and convex with respect to the topology of uniform convergence. Since the operator $T$ is continuous and maps to set $\mathcal{L}\left(L^{*}, u\right)$ into itself, it has a fixed point $F^{*}$ by Kakutani's theorem. Moreover, $g_{F^{*}}$ is a Lipschitz continuous equilibrium for our non-cooperative discounted stochastic game $\Sigma$.

Proof of Theorem 2.15: Due to Theorem 2.13 the stochastic game $\Sigma$ admits a Lipschitz continuous equilibrium $\tau$. The transition operator $K^{\tau}$ of the Markov chain $\left\{\xi_{t}\right\}$ acts on bounded measurable functions on $M$ according to (1). Since both the densities and the equilibrium strategies are Lipschitz continuous,

$$
\begin{aligned}
\lim _{n \rightarrow \infty}\left|K^{\tau} f\left(\xi_{n}\right)-K^{\tau} f(\xi)\right| & \leq \lim _{n \rightarrow \infty}\|f\|_{\infty}\left\|q\left(\xi_{n}, \tau\left(\xi_{n}\right), \eta\right)-q(\xi, \tau(\xi), \eta)\right\|_{\infty} \\
& \leq \lim _{n \rightarrow \infty} L\left(\left\|\xi_{n}-\xi\right\|_{M}+\left\|\tau\left(\xi_{n}\right)-\tau(\xi)\right\|\right)=0
\end{aligned}
$$

whenever $\lim _{n \rightarrow \infty} \xi_{n}=\xi$. In particular, the Markov chain $\left\{\xi_{t}\right\}$ has the Feller property. Thus, the existence of an initial distribution $\mu$ such that $\left\{\xi_{t}\right\}$ is stationary and ergodic under $\mathbb{P}_{\mu}^{\tau}$ follows from compactness of the state space $M$.

\subsection{Existence of Lipschitz continuous equilibria in stochastic games with unbounded state spaces}

In this section we prove our existence result for Lipschitz continuous equilibria in noncooperative stochastic games with unbounded state spaces.

Proof of Theorem 2.17: We introduce the operator $T_{n}: B_{u}\left(M_{n}, \mathbb{R}_{+}^{N}\right) \rightarrow B_{u}\left(M_{n}, \mathbb{R}_{+}^{N}\right)$ by

$$
\left(T_{n} f_{n}\right)^{i}(\xi)=\left(1-\beta^{i}\right) U^{i}\left(\xi, g_{f_{n}}(\xi)\right)+\beta^{i} \int_{M_{n}} f_{n}^{i}(\eta) q_{n}\left(\xi, g_{f_{n}}(\xi), \eta\right) \mu(d \eta)
$$

Here, $g_{f_{n}}(\xi)$ denotes the unique equilibrium in the one-shot game $\Sigma_{f_{n}}$ with average continuation function $f_{n} \in B_{u}\left(M_{n}, \mathbb{R}^{N}\right)$ and conditional densities $q_{n}$. Let $F_{n}$ be a fixed point of $T_{n}$. Due to our Lemmas 2.21 and 2.24 , the mappings $g_{F_{n}}: M_{n} \rightarrow \underline{\bar{X}}$ and $F_{n}: M_{n} \rightarrow \mathbb{R}^{N}$ $(n \in \mathbb{N})$ are Lipschitz continuous with common Lipschitz constants. In particular the sequence $\left\{\left(g_{F_{n}}, F_{n}\right)\right\}$ is equicontinuous, and so the theorem by Arzela and Ascoli yields a subsequence 
$\left(n_{k}\right)$ and Lipschitz continuous functions $F: M \rightarrow \mathbb{R}$ and $g: M \rightarrow \underline{\bar{X}}$ such that

$$
\lim _{k \rightarrow \infty}\left|F_{n_{k}}(\xi)-F(\xi)\right|=0 \text { and } \lim _{k \rightarrow \infty}\left|g_{F_{n_{k}}}(\xi)-g(\xi)\right|=0 \quad \text { uniformly on compact sets. }
$$

Since the utility functions are uniformly bounded, weak convergence of the sequence of probability measures $\left\{\mu_{n_{k}}(\xi, x ; \cdot)\right\}$ defined in $(9)$ to $\mu(\xi, x ; \cdot)$ yields

$$
\lim _{k \rightarrow \infty} \int_{H} F_{n_{k}}^{i}(\eta) q_{n_{k}}\left(\xi, g_{F_{n_{k}}}(\xi), \eta\right) \mu(d \eta)=\int_{H} F^{i}(\eta) q(\xi, g(\xi), \eta) \mu(d \eta)
$$

We deduce that

$$
F^{i}(\xi)=\left(1-\beta^{i}\right) U^{i}(\xi, g(\xi))+\beta^{i} \int_{H} F^{i}(\eta) q(\xi, g(\xi), \eta) \mu(d \eta)
$$

It is easily seen that $g(\xi)$ is an equilibrium in the one-shot game $\Sigma_{F}$ with conditional transition densities $q$. Thus, $g$ is a Lipschitz continuous Nash equilibrium in Markov strategies in the stochastic game $\Sigma$ with unbounded state space.

\section{Ergodic equilibria in stochastic games with complete con- nections}

So far we proved the existence of Nash equilibria in stochastic games which have the additional property that the equilibrium process is stationary and ergodic, given the starting point is chosen according to a suitable initial distribution. In this section we are going to extend this result for a special class of stochastic games which we shall call stochastic games with complete connection. Our goal is to formulate conditions which guarantee the existence of stationary Nash equilibria which ensure that the equilibrium process settles down in the long run. More specifically, our focus is on the existence of ergodic equilibria.

Definition 3.1 Let $\Sigma$ be a discounted non-cooperative stochastic game. We call a stationary Nash equilibrium in Markov strategies $\tau$ an ergodic equilibrium if the Markov chain $\left(\left\{\xi_{t}\right\}, \mathbb{P}_{\xi}^{\tau}\right)$ converges in distribution to a unique stationary measure $\mu^{\tau}$ on $(\Omega, \mathcal{F})$.

Clearly, an ergodic equilibrium is at the same time a stationary equilibrium; if $\mu^{\tau}$ denotes the unique limiting distribution of the state sequence, then the process $\left\{\xi_{t}\right\}$ is stationary and ergodic under the law $\mathbb{P}_{\mu^{\tau}}^{\tau}(\cdot)=\int \mathbb{P}_{\xi}^{\tau}(\cdot) \mu^{\tau}(d \xi)$. A stationary equilibrium, of course, is not necessarily ergodic. 


\subsection{Random systems with complete connections}

Before we introduce the notion of stochastic game with complete connections we recall the concept of a random system with complete connections: Let $\left(M_{1}, d_{M_{1}}\right)$ be a metric space and let $\left(M_{2}, \mathcal{M}_{2}\right)$ be a measurable space. Let $Z$ be a stochastic kernel form $M_{1}$ to $\mathcal{M}_{2}$, and let $v: M_{1} \times M_{2} \rightarrow M_{1}$ be a measurable mapping.

Definition 3.2 Following Iosefescu and Theodorescu (1968) we call the quadruple

$$
\bar{\Gamma}=\left(\left(M_{1}, d_{M_{1}}\right),\left(M_{2}, \mathcal{M}_{2}\right), Z, v\right)
$$

a homogeneous random system with complete connections (RSCC for short). Given an initial value $\xi$, a RSCC induces two stochastic processes $\left\{\xi_{t}\right\}$ and $\left\{\zeta_{t}\right\}$ on the canonical probability space $\left(\Omega, \mathcal{F}, \mathbb{P}_{\xi}\right)$ taking values in $M_{1}$ and $M_{2}$, respectively, by

$$
\xi_{t+1}=v\left(\xi_{t}, \zeta_{t}\right) \quad \text { and } \quad \mathbb{P}_{\xi}\left[\zeta_{t} \in \cdot \mid \xi_{t}, \zeta_{t-1}, \xi_{t-1}, \zeta_{t-2}, \ldots\right]=Z\left(\xi_{t} ; \cdot\right) .
$$

Here $\mathbb{P}_{\xi}\left[\xi_{0}=\xi\right]=1$. These processes are called the associated Markov chain and the signal sequence, respectively.

The following theorem summarizes results about asymptotic stability of Markov chains associated to RSCC that appear as Theorem 4.2 in Norman (1972) and as Theorem 2.1 in Barnsley, Demko, Elton, and Geronimo (1988), respectively.

Theorem 3.3 Let $\bar{\Gamma}=\left(\left(M_{1}, d_{M_{1}}\right),\left(M_{2}, \mathcal{M}_{2}\right), Z, v\right)$ be a random system with complete connections and assume that the following conditions are satisfied:

(i) The map $v: M_{1} \times M_{2} \rightarrow M_{1}$ satisfies the mean contraction condition

$$
\int_{M_{1}} d_{M_{1}}\left(v\left(\xi_{1}, \zeta\right), v\left(\xi_{2}, \zeta\right)\right) Z\left(\xi_{1} ; d \zeta\right) \leq r d_{M_{1}}\left(\xi_{1}, \xi_{2}\right)
$$

for some $r<1$ and all $\xi_{1}, \xi_{2} \in M_{1}$.

(ii) There exists a probability measure $\nu$ on $(\Omega, \mathcal{F})$ and a constant $c>0$ such that $Z(\xi ; \cdot) \geq$ $c \nu(\cdot)$ and such that

$$
\int_{M_{1}} d_{M_{1}}\left(v\left(\xi_{1}, \zeta\right), v\left(\xi_{2}, \zeta\right)\right) \nu(d \zeta) \leq r d_{M_{1}}\left(\xi_{1}, \xi_{2}\right) .
$$

Moreover, there is $L<\infty$ such that

$$
\sup _{B \in \mathcal{M}_{2}}\left|Z\left(\xi_{1} ; B\right)-Z\left(\xi_{2} ; B\right)\right| \leq L d_{M_{1}}\left(\xi_{2}, \xi_{2}\right) .
$$


Then the Markov chain $\left\{\xi_{t}\right\}$ associated to $\Gamma$ has the Feller property. If, in addition, $M_{1}$ is compact or $M_{2}$ is finite, then the sequence $\left\{\xi_{t}\right\}$ converges in distribution to a unique stationary measure $\mu$.

\subsection{Stochastic games with complete connections}

We are now ready define the notion of a stochastic game with complete connections. We define a stochastic game with complete connections as a RSCC where the associated Markov chain is under the simultaneous control of several players.

Definition 3.4 A stochastic game with complete connections (SGCC henceforth)

$$
\Gamma=\left(I,\left(M_{1},\|\cdot\|\right),\left(\underline{\bar{Y}}, U^{i}, \beta\right)_{i \in I},\left(M_{2}, \mathcal{M}_{2}\right), Z, v, \xi\right)
$$

is defined in terms of the following objects:

- $I=\{1,2, \ldots, N\}$ is a finite set of players.

- $\left(M_{1},\|\cdot\|\right)$ is a normed compact state space and $\left(M_{2}, \mathcal{M}_{2}\right)$ is a measurable space.

- $\underline{\bar{Y}}$ is a common compact and convex action space.

- $U_{i}: M_{1} \times \underline{\bar{X}} \rightarrow \mathbb{R}$ is the utility function for player $i \in I$ where $\underline{\bar{X}}:=\prod_{i \in I} \underline{\bar{Y}}$.

- $\beta \in(0,1)$ is a common discount factor.

- $Z$ is a stochastic kernel from $M_{1} \times \underline{\bar{X}}$ to $\mathcal{M}_{2}$.

- $v: M_{1} \times M_{2} \rightarrow M_{1}$ is a measurable mapping.

- $\xi \in M$ is the starting point of the state sequence.

A stochastic game with complete connections is played as follows: At each time $t \in \mathbb{N}$ the agents observe the current state $\xi_{t}$ and select, independently of each other, their action $x_{t}^{i} \in \underline{\bar{Y}}$. The selected action profile along with the current state results in the instantaneous payoff $U^{i}\left(\xi_{t}, x_{t}\right)$ to the player $i \in I$, and the new state $\xi_{t+1}$ in the following period $t+1$ is given by

$$
\xi_{t+1}=v\left(\xi_{t}, \zeta_{t}\right) \quad \text { where } \quad \zeta_{t} \sim Z\left(\xi_{t}, x_{t} ; \cdot\right)
$$


If the players are restricted to the use of stationary Markov strategies $\tau=\left(\tau^{i}\right)_{i \in I}$, then the state sequence $\left\{\xi_{t}\right\}$ may be viewed as the Markov chain associated with the RSCC

$$
\bar{\Gamma}^{\tau}=\left(\left(M_{1},\|\cdot\|\right),\left(M_{2}, \mathcal{M}_{2}\right), Z^{\tau}, v\right) \quad \text { where } \quad Z^{\tau}(\xi ; \cdot)=Z(\xi, \tau(\xi) ; \cdot)
$$

This illustrates the connection between random systems with complete connections and stochastic games with complete connections.

We are now going to combine our results about existence of Lipschitz continuous equilibria in discounted stochastic games established in the previous section with Norman's (1972) stability result for Markov chains associated to RSCC. However, we shall see that the probabilistic structure of the state sequence associated to a SGCC is more complex than the structure of the state sequences in the games analyzed so far. To simplify our analysis we will therefore assume that the law $Z(x ; \cdot)$ depends on the action profile $x \in \underline{\bar{X}}$ only through the average action. We also need to assume that the impact of an individual player on the stochastic dynamics of the state sequence is sufficiently weak.

Assumption $3.5 \quad$ (i) The measures $Z(\xi, x ; \cdot)$ on $\left(M_{2}, \mathcal{M}_{2}\right)$ take the form

$$
Z(\xi, x ; \cdot)=\hat{Z}\left(\xi, \frac{c}{N} \sum_{i=1}^{N} x^{i} ; \cdot\right)
$$

for some kernel $\hat{Z}$ from $M_{1} \times \underline{\bar{Y}}$ to $M_{2}$ and for some constant $c>0$. The law $\hat{Z}(\xi, y ; \cdot)$ has a density $z(\xi, y, \cdot)$ with respect to some measure $\mu$ on $\left(M_{2}, \mathcal{M}_{2}\right)$. The map $z$ is two times continuously differentiable and the quantities $|D z(\xi, y, \eta)|$ and $\left|D^{2} z(\xi, y, \eta)\right|$ are dominated by some functions $\varphi_{1}(\eta)$ and $\varphi_{2}(\eta)$, respectively, which are integrable with respect to $\mu$.

(ii) There exists a probability measure $\nu$ on $\left(M_{2}, \mathcal{M}_{2}\right)$ and a constant $\lambda>0$ such that $Z(\xi, x ; \cdot) \geq \lambda \nu(\cdot)$.

(iii) The map $v$ is Lipschitz continuous and satisfies the mean-contraction conditions

$$
\max \left\{\int\left\|v\left(\xi_{1}, \zeta\right)-v\left(\xi_{2}, \zeta\right)\right\| Z(\xi, x ; d \zeta), \int\left\|v\left(\xi_{1}, \zeta\right)-v\left(\xi_{2}, \zeta\right)\right\| \nu(d \zeta)\right\} \leq r\left\|\xi_{1}-\xi_{2}\right\|
$$

for some $r<1$ and for all $x \in \underline{\bar{X}}$.

(iv) The action space is a closed and convex subset of a Hilbert space and the utility functions $U^{i}$ are uniformly bounded and satisfy Assumption 2.6. 
(v) The Moderate Social Influence condition holds.

We are now ready to prove that a stochastic game with complete connections in which the impact of an individual player on the dynamics of the state sequence is weak enough has an ergodic equilibrium.

Theorem 3.6 Let $\Sigma$ be a stochastic game with complete connections that satisfies Assumption 3.5. There exists $C^{*}>0$ such that, for all $c<C^{*}$, the game $\Sigma$ has an ergodic equilibrium.

Proof: Let us assume that the stochastic game with complete connections $\Gamma$ admits a Nash equilibrium in Markov strategies $\tau^{*}$ that depends in a Lipschitz continuous manner on the current state. In this case it is enough to show that the random system $\bar{\Gamma}^{\tau^{*}}$ defined by (21) satisfies the assumptions of Theorem 3.3. For this, it suffices to show that there is a constant $\hat{L}$ so that

$$
\sup _{B \in \mathcal{M}_{2}}\left|Z^{\tau^{*}}\left(\xi_{1} ; B\right)-Z^{\tau^{*}}\left(\xi_{2} ; B\right)\right| \leq \hat{L}\left\|\xi_{1}-\xi_{2}\right\| .
$$

This, however, follows from (21) and from Lipschitz continuity of $\tau^{*}$. If $L^{*}$ denotes the Lipschitz constant of $\tau^{*}$, then

$$
\sup _{B \in \mathcal{M}_{2}}\left|Z^{\tau^{*}}\left(\xi_{1} ; B\right)-Z^{\tau^{*}}\left(\xi_{2} ; B\right)\right| \leq L\left(1+L^{*}\right)\left\|\xi_{1}-\xi_{2}\right\|
$$

due to Assumption 3.5 (i). Thus, it remains to show that $\Sigma$ has a Lipschitz continuous equilibrium in Markov strategies.

By analogy with our previous considerations, we introduce the reduced one-shot game $\Gamma_{f}$ with payoff functions

$$
U^{i, f}(\xi, x)=\left(1-\beta^{i}\right) U^{i}(\xi, x)+\beta^{i} \int_{M_{2}} f^{i} \circ v(\xi, \zeta) z(\xi, x, \eta) \nu(d \zeta)
$$

Using the same arguments as in the proof of Lemma 2.21 (iii) one can easily show that $\Sigma_{f}$ has a unique equilibrium $g_{f}(\xi)$. However, since the integral term in (23) depends on the current state $\xi$ both through $z(\xi, \cdot)$ and through $f_{i} \circ v(\xi, \cdot)$, the map $g_{f}$ typically will not be Lipschitz continuous uniformly in the average continuation function $f \in B_{u}\left(M, \mathbb{R}^{N}\right)$.

Instead of working with $B_{u}\left(M, \mathbb{R}^{N}\right)$, let us introduce the class $\mathcal{L}_{\widetilde{L}, u}\left(M, \mathbb{R}^{N}\right)$ of all Lipschitz continuous function $f: M \rightarrow \mathbb{R}^{M}$ which are Lipschitz continuous with constant $\widetilde{L}$ and satisfy $\left\|f^{i}\right\|_{\infty} \leq u^{i}$. Applying the same arguments as in the proof of Lemma 2.21 (ii) and (iv) and 
taking into account Assumption 3.5 (i) and (ii), the fact that $f$ is Lipschitz with constant $\widetilde{L}$ and because $v$ is Lipschitz continuous we obtain $c_{1}$ and $c_{2}$ such that

$$
\left\|g_{f}^{i}\left(\xi_{1}\right)-g_{l}^{i}\left(\xi_{2}\right)\right\|_{i} \leq c \frac{c_{1} \widetilde{L}+c_{2}}{N}\left\|\xi_{1}-\xi_{2}\right\|_{M}
$$

Thus, there is a constant $c_{3}$ such that the operator $T$ defined by

$$
T f_{i}(\xi)=\left(1-\beta^{i}\right) U^{i}\left(\xi, g_{f}(\xi)\right)+\beta \int_{M_{2}} f^{i} \circ v(\xi, \zeta) z\left(\xi, g_{f}(\xi), \eta\right) \nu(d \zeta)
$$

maps $\mathcal{L}_{\widetilde{L}, u}\left(M, \mathbb{R}^{N}\right)$ continuously into $\mathcal{L}_{L^{*}(\widetilde{L}), u}\left(M, \mathbb{R}^{N}\right)$ where $L^{*}(L)$ is of the form

$$
L^{*}(\widetilde{L})=c_{3}\left(1+c \frac{c_{1} \widetilde{L}+c_{2}}{N}\right) .
$$

Thus, choosing $C^{*}=\frac{N}{c_{1} c_{3}}$ we see that, for all $c<C^{*}$, there exists a sufficiently large $\hat{L}$ such that $L^{*}(\hat{L})<\hat{L}$. In particular, $T$ maps to compact set $\mathcal{L}_{\hat{L}, u}\left(M, \mathbb{R}^{N}\right)$ continuously into itself and therefore has a fixed point by Brower's theorem. This yields the existence of a Lipschitz continuous equilibrium in Markov strategies by analogy to the proof of Theorem 2.13. For more detailed arguments we refer the reader to Horst (2002).

\section{A toy model for asset pricing with strategic interaction}

This section illustrates how stochastic games with complete connections arise in simple microstructure models for financial markets. At the same time we are going to show how strategic interaction between different market participants creates an additional source of uncertainty in financial market models.

Standard financial market models usually assume identical investors who all share the same rational expectations of a future asset price, and who instantaneously and rationally discount all market information into the present price. From this academic point of view, large price shifts reflect rational changes in the valuation of an asset rather than irrational swings in the sentiment of investors. Traders, by contrast, often consider markets as being less rational. Many believe that technical trading is possible, that some kind of "market psychology" exists, and that herd effects unrelated to economic fundamentals can cause bubbles or crashes. In view of such market realities there has recently been an increasing interest in microstructure models for financial markets; see, e.g., Brock and Hommes (1997), Föllmer and Schweizer 
(1993) or Föllmer, Horst, and Kirman (2002). Most of these models regard asset prices as the result of an interaction between many small agents with bounded rationality. Our aim is to introduce an additional strategic component into the interaction.

Following Horst (2000), we consider a financial market model with an infinite set $\mathbb{A}$ of economic agents trading a single risky asset. In reaction to a proposed price $p$ in period $t \in \mathbb{N}$ the agent $a \in \mathbb{A}$ forms the excess demand

$$
e_{t+1}^{a}(p, \omega):=\ln \hat{S}_{t+1}^{a}(\omega)-\ln p
$$

where $\hat{S}_{t+1}^{a}(\omega)$ denotes his random individual reference level for the following period $t+1$. For simplicity, we assume $\log$ linear benchmarks. More precisely, $\ln \hat{S}_{t+1}^{a} \in\left\{R_{t+1}^{0}, R_{t+1}^{1}\right\}$ where

$$
R_{t+1}^{i}=\ln S_{t}+\gamma\left(F^{i}-\ln S_{t}\right) \quad\left(F^{0}<F^{1}\right)
$$

for some $\gamma<1$. Thus, the agent $a \in \mathbb{A}$ can choose between two fundamentalist benchmarks in the sense of Föllmer et al. (2002). We refer the interested reader to Föllmer et al. (2002) and the references therein for microstructure models which take trend chasing and learning effects into account. In our simple toy model $S_{t} \in\left[F^{0}, F^{1}\right]$, and so we may with no loss of generality assume that $F^{0}=0$ and that $F^{1}=1$.

We denote by $y_{t}^{a} \in\{0,1\}$ the individual choice of agent $a \in \mathbb{A}$ at time $t \in \mathbb{N}$. That is, $y_{t}^{a}=i$ if $\hat{S}_{t+1}^{a}=R_{t+1}^{i}$. The configuration of individual states in period $t$ is denoted $y_{t}=\left(y_{t}^{a}\right)_{a \in \mathbb{A}}$ and to $y_{t}$ we associate the fraction

$$
\pi\left(y_{t}\right)=\lim _{n \rightarrow \infty} \frac{1}{\left|\mathbb{A}_{n}\right|} \sum_{a \in \mathbb{A}_{n}} y_{t}^{a}
$$

of "optimistic" agents if the limit exists along a fixed increasing sequence of finite sets $\mathbb{A}_{n} \uparrow \mathbb{A}$. The actual stock price is now determined by the market clearing condition of zero total excess demand, and so the logarithmic asset price process is described by the stochastic sequence $\left\{S_{t}\right\}$ defined recursively by the linear relation

$$
S_{t+1}=(1-\gamma) S_{t}+\gamma \pi\left(y_{t}\right)
$$

Remark 4.1 Observe that the evolution of the average action is the only component affecting the formation of equilibrium prices.

Let us now specify the dynamics of the microscopic process $\left\{y_{t}\right\}$ which described the stochastic evolution of all the individual states. We wish to allow for imitation and contagion 
effects in the formation of agents' expectation, and so we assume that an agent $a \in \mathbb{A}$ chooses his new state $y_{t+1}^{a}$ at random according to a probability law which depends on (i) his current state $y_{t}^{a}$, (ii) on some signal $s_{t} \in[0,1]$ about the actual mood of the market $\pi\left(y_{t}\right)$ and (iii) on a signal $m_{t} \in[0,1]$ that depends on the actions $x_{t}=\left(x_{t}^{1}, x_{t}^{2}, \ldots, x_{t}^{N}\right) \in[0,1]^{N}$ taken by some big players. The conditional laws $K\left(\pi\left(y_{t}\right) ; \cdot\right)$ and $Q\left(x_{t} ; \cdot\right)$ of the signals $s_{t}$ and $m_{t}$, given the current $\operatorname{mood} \pi\left(y_{t}\right)$ and the action profile $x_{t}$, respectively, are described by the respective stochastic kernels $K$ on $[0,1]$ and $Q$ from $[0,1]^{N}$ to $[0,1]$. Given $\left(s_{t}, m_{t}\right)$, the agent $a \in \mathbb{A}$ chooses his new state according to the probability distribution $\pi_{s_{t}, m_{t}}\left(y_{t}^{a} ; \cdot\right)$ on $\{0,1\}$. The dynamics of the microscopic process $\left\{y_{t}\right\}$ therefore is described by a Markov chain

$$
\Pi_{s_{t}, m_{t}}\left(y_{t} ; \cdot\right)=\prod_{a \in \mathbb{A}} \pi_{s_{t}, m_{t}}\left(y_{t}^{a} ; \cdot\right)
$$

in the random environment $\left\{\left(s_{t}, m_{t}\right)\right\}$, and the evolution of the sequence $\left\{\left(s_{t}, m_{t}\right)\right\}$ is partially controlled by the big players. If the average action $\pi\left(y_{t}\right)$ exists, the law of large numbers for i.i.d. random variables yields

$$
\pi\left(y_{t+1}\right)=v\left(\pi\left(y_{t}\right), s_{t}, m_{t}\right):=\pi_{s_{t}, m_{t}}(1 ; 1) \pi\left(y_{t}\right)+\pi_{s_{t}, m_{t}}(-1 ; 1)\left(1-\pi\left(y_{t}\right)\right)
$$

for $\Pi_{s_{t}, m_{t}}\left(y_{t} ; \cdot\right)$-a.e. $y_{t+1}$. Thus, the sequence $\left\{\pi\left(y_{t}\right)\right\}$ exists almost surely if $\pi\left(y_{0}\right)$ exists. Let us now assume that the big players wish to control to evolution of the mood of the market and, thereby, also the dynamics of stock prices. In view of (25), the process $\left\{\pi\left(y_{t}\right)\right\}$ may be viewed as the state sequence associated to the stochastic game with complete connections

$$
\Gamma=\left(I,[0,1],\left([0,1], U^{i}, \beta\right)_{i \in I},[0,1]^{2}, Z, v\right)
$$

Here the map $v:[0,1]^{3} \rightarrow[0,1]$ is given by $(25)$, the stochastic kernel $Z$ from $[0,1] \times[0,1]^{N}$ to $[0,1]^{2}$ takes the form

$$
Z(\pi(y), x ; \cdot)=K(\pi(y) ; \cdot) \otimes Q(x ; \cdot)
$$

and the utility functions $U^{i}:[0,1] \times[0,1]^{N} \rightarrow \mathbb{R}$ are bounded and two times continuously differentiable.

One can now show that $v(s, m ; \cdot)$ is a contraction on $[0,1]$ uniformly in $(s, m) \in[0,1]^{2}$ if $0<\pi\left(y^{a} ; 1\right)<1$. Thus, if the assumption of Theorem 3.6 are satisfied, i.e., if the impact of an individual big player on the choices of the small investors is sufficiently weak, then there exists an equilibrium strategy $\tau$ for the big players such that the mood of the market settles down in the long run. Given $\tau$, the process $\left\{\left(S_{t}, \pi\left(x_{t}\right)\right)\right\}$ may be viewed as the Markov chain 
on $[0,1]^{2}$ associated to a suitable random system with complete connections that satisfies the assumptions of Theorem 3.3. In particular, there exists a unique measure $\mu^{\tau}$ such that the price process converges in law to $\mu^{\tau}$. In this sense the asymptotic behavior of asset prices is uniquely determined, conditioned on the equilibrium strategy $\tau$. In contrast to microstructure models for financial markets without strategic interaction, however, there is no reason to assume that the asymptotic behavior of the stock price process is uniquely determined. In this sense, strategic interaction between different market participants may be viewed as an additional source of uncertainty in financial markets.

\section{Conclusion}

We established the existence of stationary Markov equilibria for a class of stochastic games with compact state and action spaces and norm-continuous transition probabilities. Our proof is based on the observation that infinite horizon discounted stochastic games in which the interaction between different players is not too strong have equilibria in Markov strategies that depend in a Lipschitz continuous manner on the current state. For the special class of stochastic games with complete connections this yields the existence of ergodic equilibria, i.e., of equilibria which guarantee that the equilibrium process settles down in the long run. Such games arise in microstructure models for financial markets in which they provide a framework for analyzing the evolution of asset prices in the presence of strategically interacting agents.

\section{References}

Amir, R. (1996a): "Continuous Stochastic Games of Capital Accumulation with Convex Transitions," Games and Economic Behavior, 15, 111-131.

- (1996b): "Cournot Oligopoly and the Theory of Supermodular Games," Games and Economic Behavior, 15, 132-148.

Barnsley, M. F., S. G. Demko, J. H. Elton, and J. S. Geronimo (1988): "Invariant Measures for Markov Processes Arising from Iterated Function Systems with PlaceDependent Probabilities," Ann. Inst. Henri Poincare, 24, 367-394.

Brock, W. A., And C. Hommes (1997): "A rational route to randomness," Econometrica, $65(5), 1059-1095$. 
Curtat, L. (1996): "Markov equilibria in stochastic games with complementaries," Games and Economic Behavior, 17, 177-199.

Diamond, P. (1985): "Aggregate Demand Management in Search Equilibrium," J. Political Economy, 90, 881-894.

Duffie, D., J. Geanakopolos, A. MasColell, and A. Mclennan (1994): "Stationary Markov Equilibria," Econometrica, 62 (4), 745-781.

Federgruen, A. (1978): "On N-Person Stochastic Games with Denumerable State Space," Adv. Appl. Probab., 10, 452-471.

Föllmer, H., U. Horst, ANd A. Kirman (2002): "On the transitory nature of gurus," Working Paper, Princeton University.

Föllmer, H., And M. Schweizer (1993): "A Microeconomic Approach to Diffusion Models for Stock Prices," Mathematical Finance, 3, 1-23.

Guesnerie, R., And M. Woodford: "Endogeneous Fluctuations," in Advances in Economic Theory, Volume II, ed. by J.J. Laffont. Cambridge, Cambridge University Press.

Horst, U. (2000): "Asymptotics of Locall and Globally Interacting Markov Chains Arising in Microstructure Models for Financial Markets", PhD-Thesis. Humboldt-University of Berlin.

(2002): "Stability of Linear Stochastic Difference Equations in Controlled Random Environments," Working Paper. Humboldt-University of Berlin.

Horst, U. And, J. Scheinkman (2002): "Equilibria in systems of random social interactions," Working paper. Princeton University.

Iosefescu, M., And M. Theodorescu (1968): Random Processes and Learning. SpringerVerlag, Berlin.

KÜEnLE, H.U. (1999): "Equilibrium Strategies in Stochastic Games with Additive Cost and Transition Structure," International Game Theory Review, 1, 131-147.

Mertens, F. And, T. Parthasarathy (1987): "Equilibria for discounted stochastic games," CORE Discussion Paper 8750, Leuven, Universite Catholique de Louvian. 
Milgrom, P., And J. Roberts (1990): "Rationalizability, Learning, and Equilibrium in Games with Strategic Complementarities," Econometrica, 58 (6), 1255-1277.

Montrucchio, L. (1987): "Lipschitz continuous policy functions for strongly concave optimization problems," Journal of Mathematical Economics, 16, 259-273.

Norman, F. M. (1972): Markov Processes and Learning Models. Academic Press, New York.

NowaK, A. (1985): "Existence of equilibrium stationary strategies in discounted noncooperative stochastic games with uncountable state space," J. Optim. Theory Appl., 45, $591-602$.

NowaK, A. (1987): "Nonrandomized Strategy Equilibria in Non-Cooperative Stochastic Games with Additive Transition and Reward Structure," J. Optim. Theory Appl., 52, $429-441$.

Parthasarathy, T. (1982): "Existence of Equilibrium Stationary Strategies in Discounted Stochastic Games," Sankya, 44, 114-127.

Parthasarathy, T., AND S. Sinha (1989): "Existence of Stationary Equilibrium Strategies in Non-Zero Sum Discounted Stochastic Games with Uncountable State Space and State Independent Transitions," International Journal of Game Theory, 18, 189-194.

Shapley, L. (1953): "Stochastic Games," Proceedings of the National Academy of Sciences, USA, 39, 1095-1100.

TOPKIS, D. (1978): "Minimizing a supermodular function on a lattice," Operations Research, $26,305-321$.

Topkis, D. (1998): Supermodularity and Complementarity. Princeton Academic Press, Princeton.

Vival, J. P. (1983): "Strong and Weak Convexity of Sets and Functions," Mathematics of Operations Research, 8 (2), 231-259. 